\title{
Giant Depolarizing Potentials: the Septal Pole of the Hippocampus Paces the Activity of the Developing Intact Septohippocampal Complex In Vitro
}

\author{
Xavier Leinekugel, Ilgam Khalilov, Yehezkel Ben-Ari, and Roustem Khazipov \\ Institut National de la Santé et de la Recherche Médicale, 75014 Paris, France
}

In neonatal hippocampal slices, recurrent spontaneous giant depolarizing potentials (GDPs) provide neuronal synchronized firing and $\mathrm{Ca}^{2+}$ oscillations. To investigate the possible role of GDPs in the synchronization of neuronal activity in intact neonatal limbic structures, we used multiple simultaneous electrophysiological recordings in the recently described preparation of intact neonatal septohippocampal complex in vitro. Combined whole-cell (in single or pairs of cells) and extracellular field recordings (one to five simultaneous recording sites) from the CA3 hippocampal region and various parts of the septum indicated that spontaneous GDPs, which can be initiated anywhere along the longitudinal hippocampal axis, are most often initiated in the septal poles of hippocampus and propagate to medial septum and temporal poles of both hippocampi simultaneously. GDPs were abolished in the medial septum but not in the hippocampus after surgical separation of both structures,

Synchronized and propagating neuronal activities shape neuronal pathways during the early stages of development. Neuronal synchronization during development can be provided by sensory experience, as well as by endogenous patterns of activity (O’Donovan et al., 1992; Yuste et al., 1992, 1995; Gu and Spitzer, 1995; Kandler and Katz, 1995; Feller et al., 1996; Ben-Ari et al., 1997), and participate in network formation by activitydependent mechanisms (Constantine-Paton et al., 1990; Goodman and Shatz, 1993; Katz and Shatz, 1996). It is therefore of primary importance to describe whether and how spontaneous patterns coordinate the activity of neurons inside and across intact developing neuronal structures.

Limbic structures form a functional complex in the adult and are implicated in memory processes and pathological disorders. Although physiological interactions and associated patterns of activity (theta rhythm, sharp waves) between septum and hippocampus have been extensively studied in the adult (for review, see Buzsaki and Chrobak, 1995; Freund and Buzsaki, 1996), little is known about the spontaneous patterns of activity present in these structures at the early stages of development. Previous works in the rat hippocampal slice preparations have shown that

\footnotetext{
Received March 3, 1998; revised June 8, 1998; accepted June 9, 1998.

This work was funded by Institut National de la Santé et de la Recherche Médicale (I.K.), Ministere de l'Education Nationale, and Foundation pour la Recherche Medicale (X.L.). We thank Dr. V. Dzhala for useful comments and discussions, Dr. M. Esclapez for help in morphological analysis, and S. Weiller for technical assistance.

Correspondence should be addressed to Xavier Leinekugel, Institut National de la Santé et de la Recherche Médicale U.29, 23, Boulevard de Port Royal, 75014 Paris, France. E-mail: leinekugel@cochin.inserm.fr

Copyright (ㄷ) 1998 Society for Neuroscience $\quad 0270-6474 / 98 / 186349-09 \$ 05.00 / 0$
}

suggesting hippocampal origin of GDPs. The preferential septotemporal orientation of GDP propagation observed in the intact hippocampus was associated with a corresponding gradient of GDP frequency in isolated portions of hippocampus. Accordingly, most GDPs propagated in the septotemporal direction in both septal and temporal hippocampal isolated halves, and whereas GDP frequency remained similar in the septal part of hippocampus after its surgical isolation, it progressively decreased in more temporally isolated portions of the hippocampus. Because GDPs provide most of the synaptic drive of neonatal neurons, they may modulate the development of neuronal connections in the immature limbic system.

Key words: giant depolarizing potentials (GDPs); GABAergic network; synchronized neuronal activity; oscillations in neonates; rat; development; intact neonatal hippocampus and septum in vitro; electrophysiology

during the first postnatal week [postnatal days 0-5 (P0-P5)], the spontaneous neuronal activity is characterized by network-driven giant depolarizing potentials (GDPs) (Ben-Ari et al., 1989). In slices, GDPs provide synchronous neuronal activation and $\mathrm{Ca}^{2+}$ oscillations attributable to the cooperation of excitatory GABAergic and glutamatergic synaptic transmissions (Corradetti et al., 1988; Gaïarsa et al., 1990; Leinekugel et al., 1995, 1997a, 1998; Ben-Ari et al., 1997; Khazipov et al., 1997). Understanding the coordination of neuronal activity in the intact neonatal septohippocampal system is a major requirement for the study of activity-dependent processes implicated in the formation of functional neuronal ensembles in the limbic system. We presently investigated whether and how GDPs coordinate neuronal activity in the intact neonatal septohippocampal complex in vitro.

A major obstacle for the study of generation and propagation of organized neuronal activities in the brain is that they may require intact neuronal networks. In vivo recordings, which preserve neuronal networks intact, do not offer the technical facilities of in vitro experiments, can hardly be made in neonates because of intrinsic difficulties, and the use of anesthetics may affect synaptic transmission (Mooney et al., 1996). On the other hand, more complex in vitro preparation than slices in which the neuronal network is damaged are clearly required to study the generation and propagation of synchronized neuronal activities. A successful development was recently realized in the study of neuronal activity propagation in the visual system: using a preparation including the intact retina and visual pathway to LGN slices from neonatal mice in vitro, Mooney et al. (1996) observed that spontaneous waves of activity in the retina before the onset of vision propagated to LGN, which could play a crucial role in the forma- 
tion of functional transmission of subsequent visual information. We have shown recently that neuronal activity in neonatal rat intact limbic structures (including both hippocampi and septum functionally connected) could also be studied in vitro (Khalilov et al., 1997a,b; Leinekugel et al., 1997b). The use of multiple simultaneous patch-clamp and extracellular field recordings in this novel in vitro preparation enabled us to observe that GDPs provide synchronized neuronal activity in the intact neonatal septohippocampal complex and support a septotemporal orientation of hippocampal activity. Because GDPs provide most of the synaptic drive of neonatal neurons, they may modulate the development of neuronal connections in the immature limbic system.

\section{MATERIALS AND METHODS}

Preparation of acute intact hippocampi. The intact septohippocampal complexes or intact hippocampal formations (IHFs) were prepared as described previously (Khalilov et al., 1997a). Neonatal male Wistar rats (age, P0-P6) were anesthetized by hypothermia and decapitated. After decapitation, the brains were quickly removed and immersed for dissection in ice-cold $\left(2-4^{\circ} \mathrm{C}\right)$ oxygenated standard artificial CSF (ACSF) composed of (in $\mathrm{mm}$ ): $126 \mathrm{NaCl}, 3.5 \mathrm{KCl}, 2.0 \mathrm{CaCl}_{2}, 1.3 \mathrm{MgCl}_{2}, 25$ $\mathrm{NaHCO}_{3}, 1.2 \mathrm{NaH}_{2} \mathrm{PO}_{4}$, and 11 glucose). After removing the cerebellum and the most frontal part of the neocortex by coronal sectioning, the complex including the two hippocampi and septum was gently isolated from surrounding structures with two plastic spatulas. Great care was given to avoid damage of connections between structures. For a number of experiments, single IHFs were then isolated from the septohippocampal complex. The septohippocampal complex or the IHFs were then gently transferred to a beaker containing oxygenated ACSF and kept at room temperature $\left(20-22^{\circ} \mathrm{C}\right)$ for at least $1-2 \mathrm{hr}$ before use. They were then transferred to a fully submerged chamber, laid on a nylon mesh, fixed with entomological needles inserted to Sylgard, and superfused at a rate of $10-12 \mathrm{ml} / \mathrm{min}$ with oxygenated $\mathrm{ACSF}\left(30-32^{\circ} \mathrm{C}\right)$. The preparation was laid down on its internal side to allow direct access to the CA1 and $\mathrm{CA} 3$ regions.

Electrophysiological recordings. Electrophysiological recordings were performed using the patch-clamp technique in the whole-cell configuration (Blanton et al., 1989) with Axopatch 200 (Axon Instruments) patchclamp amplifiers. Cells were patched blindly with 7-10 M $\mathrm{M}$ microelectrodes containing one of the following (in $\mathrm{mM}$ ): (1) $120 \mathrm{Cs}$ gluconate, 10 $\mathrm{CsCl}, 1 \mathrm{CaCl}_{2}, 10 \mathrm{EGTA}, 2 \mathrm{MgATP}$, and $10 \mathrm{HEPES}$; (2) $135 \mathrm{~K}$ gluconate, $2 \mathrm{MgCl}_{2}, 0.1 \mathrm{CaCl}_{2}, 2 \mathrm{Na} 2 \mathrm{ATP}, 1 \mathrm{EGTA}$, and $10 \mathrm{HEPES}$; or (3) $140 \mathrm{CsCl}, 1 \mathrm{CaCl}_{2}, 10 \mathrm{EGTA}, 10 \mathrm{HEPES}$, and $2 \mathrm{MgATP}, \mathrm{pH} 7.25$; osmolarity, 270-280 mOsm. Lucifer yellow (0.2-0.4\%) was routinely added to the pipette solution for post hoc morphological identification.

Extracellular field potentials were recorded conventionally using glass micropipettes filled with ACSF (1-5 M $\Omega$ ) and DAM80 amplifiers (low filter, $1 \mathrm{~Hz}$; high filter, $0.1 \mathrm{KHz}$; WPI). Electrical stimulations $(0-80 \mathrm{~V}$, $10-30 \mu \mathrm{sec})$ were provided by a bipolar electrode placed in the CA3 hippocampal region.

Data analysis. Spontaneous neuronal activity and stimulation-evoked responses were acquired on a digital audio tape recorder (Biologic) and loaded into an 80486 personal computer using an analog-to-digital converter (TL1 DMA; Labmaster). Acquis (sampling rate, $1.3 \mathrm{KHz}$; Axon) and Axotape (sampling rate, $0.5 \mathrm{KHz}$; Axon) programs were used for the acquisition and analysis of evoked and spontaneous events, respectively.

Group measures were expressed as mean \pm SEM. Statistical significance of differences between means was assessed with the Student's $t$ test with the aid of the statistical software StatView SE+ Graphics (Abacus Concepts, Calabases, CA). The level of significance was set at $p<0.05$.

Drugs. Tetrodotoxin was purchased from Sigma (St. Louis, MO); bicuculline, CNQX, and APV were purchased from Tocris Neuramin; and Lucifer yellow was purchased from Molecular Probes (Eugene, OR).

\section{RESULTS}

Spontaneous synaptic activity in the limbic system of newborn rats was investigated using electrophysiological techniques combining patch-clamp and extracellular field-potential recordings in the in vitro preparation containing septum and both hippocampi dissected from P0-P6 animals.

\section{Synaptic activity of individual neurons in the intact septum and hippocampus is characterized by network-driven GDPs}

Patch-clamp whole-cell recordings were performed blindly in the CA3 region of hippocampus and medial septum from 19 animals. The addition of Lucifer yellow in the internal solution allowed for post hoc identification of the recorded neurons.

As in age-matched hippocampal slices, the spontaneous activity of hippocampal neurons ( $n=104$ cells from 22 hippocampi) was characterized by recurrent GDPs (Ben-Ari et al., 1989). GDPs are network-driven events: (1) their amplitude, but not frequency, was modified by changing the membrane potential (Fig. 1A); (2) they were reversibly blocked by perfusion with high- $\mathrm{Mg}^{2+}(9 \mathrm{mM})$ external solution (data not shown); and (3) they were evoked by electrical stimulation in an all-or-none manner, increasing stimulation intensity above threshold decreasing their latency without modifying their amplitude (Fig. 1B). GDPs in the intact hippocampus had features similar to those described in slices: (1) they were reversibly blocked by bath application of TTX $(1 \mu \mathrm{M})$ or the $\mathrm{GABA}_{\mathrm{A}}$ and glutamate receptor antagonists bicuculline (10 $\mu \mathrm{M})$, APV $(50 \mu \mathrm{M})$, and CNQX $(10 \mu \mathrm{M})$, respectively (data not shown), confirming that they are mediated by synaptic transmission via the activation of $\mathrm{GABA}_{\mathrm{A}}$ and glutamate receptors (BenAri et al., 1997; Khazipov et al., 1997; Leinekugel et al., 1997a); and (2) the reversal potential of the GDPs was highly dependent on internal $\mathrm{Cl}^{-}\left(4.2 \mathrm{mM} \mathrm{Cl}^{-}, E_{\mathrm{inv}}=-57 \pm 2 \mathrm{mV} ; n=17 ; 12 \mathrm{mM}\right.$ $\mathrm{Cl}^{-}, E_{\mathrm{inv}}=-47 \pm 2 \mathrm{mV} ; n=4 ;$ and $142 \mathrm{mM} \mathrm{Cl}^{-}, E_{\mathrm{inv}}=-2 \pm$ $5 \mathrm{mV} ; n=8$ ), indicating that they were primarily mediated by $\mathrm{GABA}_{\mathrm{A}}$ receptors (Fig. $1 C$ ).

Interestingly, GDPs were present also in neurons from the medial septum with similar properties $(n=15)$ (Fig. $1 D)$, suggesting that they represent a common pattern of neuronal activity in developing interconnected limbic structures. Because GDPs in hippocampal transverse slices were shown to provide synchronous neuronal activity (Khazipov et al., 1997; Leinekugel et al., 1997a), we investigated whether neuronal activity among these developing limbic structures was synchronized.

\section{GDPs are locally synchronized events that propagate}

As a first approach to study GDP synchronization, we combined whole-cell and extracellular field potential recordings at different locations in the intact hippocamposeptal complex. An extracellular electrode was placed in the CA3 pyramidal region of hippocampus or in medial septum, and a nearby individual neuron was simultaneously recorded in the whole-cell mode. As illustrated in Figure 1, $C$ and $D$, GDPs in individual cells were highly synchronized with local extracellular field potentials, suggesting that GDPs are locally synchronized events $(n=6$ whole-cell-field pairs in hippocampus; $n=8$ whole-cell-field pairs in septum). Moreover, as illustrated in Figure 2, double patch-clamp recordings ( $n=12$ pairs) indicated that GDPs propagate from one hippocampus to the other: (1) GDPs, which are typically associated with a burst of one to five action potentials (K hazipov et al., 1997; Leinekugel et al., 1997a), were synchronous in CA3 neurons from both hippocampi (Fig. $2 B-D$ ); and (2) electrical stimulation in one hippocampus evoked the generation of ipsilateral and contralateral GDPs (Fig. 2E,F).

In keeping with these results, both spontaneous and evoked GDPs were highly synchronous in simultaneous whole-cell recordings from pairs of individual neurons close to each other $(n=$ 32 pairs). Spontaneous GDPs also appeared synchronously in pairs of neurons comprising one neuron in each hippocampus at 
A
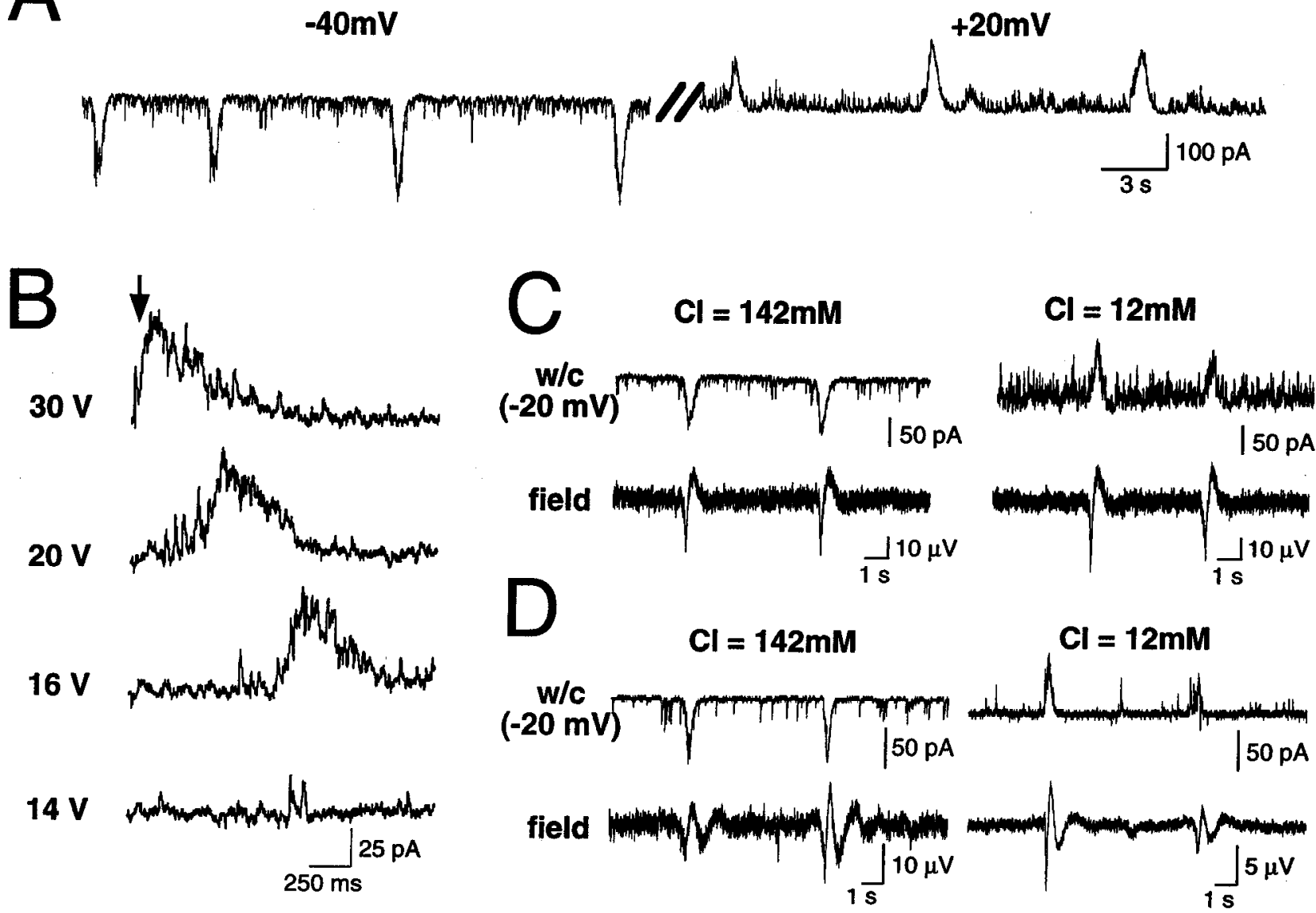

Figure 1. Spontaneous and evoked GDPs are present in neonatal hippocampal and medioseptal neurons of the intact septohippocampal complex in vitro. $A$, Whole-cell recording (voltage clamp) at two different membrane potentials (left, $-40 \mathrm{mV} ;$ right, $+20 \mathrm{mV}$ ) from a CA3 pyramidal cell. Note the presence of recurrent GDPs which amplitude, but not frequency, changed with membrane potential. B, GDPs evoked by electrical stimulations (arrow) in a CA3 pyramidal cell. Note that above threshold, increasing stimulation decreases GDP latency without affecting its amplitude. $C-D$, Whole-cell recordings with different internal $\mathrm{Cl}^{-}$concentrations (top traces: left, $\mathrm{Cl}_{\mathrm{i}}=142 \mathrm{mM} ;$ right, $\mathrm{Cl}_{\mathrm{i}}=12 \mathrm{mM}$ ) and extracellular field recordings (bottom traces) of spontaneous GDPs in the CA3 region of hippocampus $(C)$ and in the medial septum $(D)$. GDPs were synchronized in individual neurons $(w / c$, whole cell) and in the local neuronal population ( field, field recordings very close to whole-cell pipette). Note also that GDP amplitude strongly depends on internal $\mathrm{Cl}^{-}$in neurons from both hippocampus and medial septum.

similar distances from their respective septal poles ( $n=12$ pairs) (Fig. 2B-D). However, with recordings from distant cells along the septotemporal axis, significant delays of up to $1 \mathrm{sec}$ were systematically observed. In fact, whereas evoked GDPs appeared first in the cell closest to the stimulus location (in both single and bilateral hippocampi) (Fig. 2F), spontaneous GDPs appeared most often first in the cell closest to the septal pole of hippocampus. These results suggest that locally synchronized spontaneous GDPs initiate in the septal poles of the hippocampus and then propagate toward the temporal poles.

To directly address this question, simultaneous multiple fieldpotential recordings were used, allowing us to study the origin and propagation of GDPs in the neonatal septohippocampal complex.

\section{Spontaneous GDPs originate in the septal poles of hippocampus and propagate to temporal poles}

Three to five extracellular electrodes for field recordings were placed in the CA3 region at various locations along the septotemporal axis of one or both hippocampi. Each experiment consisted in the recording of 50-200 consecutive GDPs. As illustrated in
Figure 3, GDPs appeared synchronously in both hippocampi and propagated from septal to temporal poles. In average of 28 experiments, $82 \pm 3 \%$ of GDPs appeared in the most septal recording site and propagated in the septotemporal direction, whereas $3 \pm 2 \%$ appeared in the middle of hippocampus and propagated in both septal and temporal directions, $5 \pm 2 \%$ appeared in the temporal pole and propagated toward the septal pole, $5 \pm 1 \%$ appeared simultaneously in all recording sites, and $5 \pm 1 \%$ appeared in only one recording site and did not propagate. These features were very similar when recordings were obtained from two interconnected hippocampi $(n=13)$ (Fig. 3) or in the isolated hippocampus $(n=15)$ (Fig. $4 A)$. Most GDPs recorded in one hippocampus also appeared in the contralateral one ( $84 \pm 9 \% ; n=8)$. Although GDPs tended to appear first in the left hippocampus, no significant leading behavior between left versus right septal hippocampal poles was evidenced; $18 \pm 4 \%$ of GDPs appeared synchronously in both septal poles, $54 \pm 9 \%$ appeared first in the left hippocampus, and $28 \pm 9 \%$ appeared first in the right hippocampus $(n=8 ; p=0.2)$.

Interestingly, a limited proportion of GDPs appeared in both 


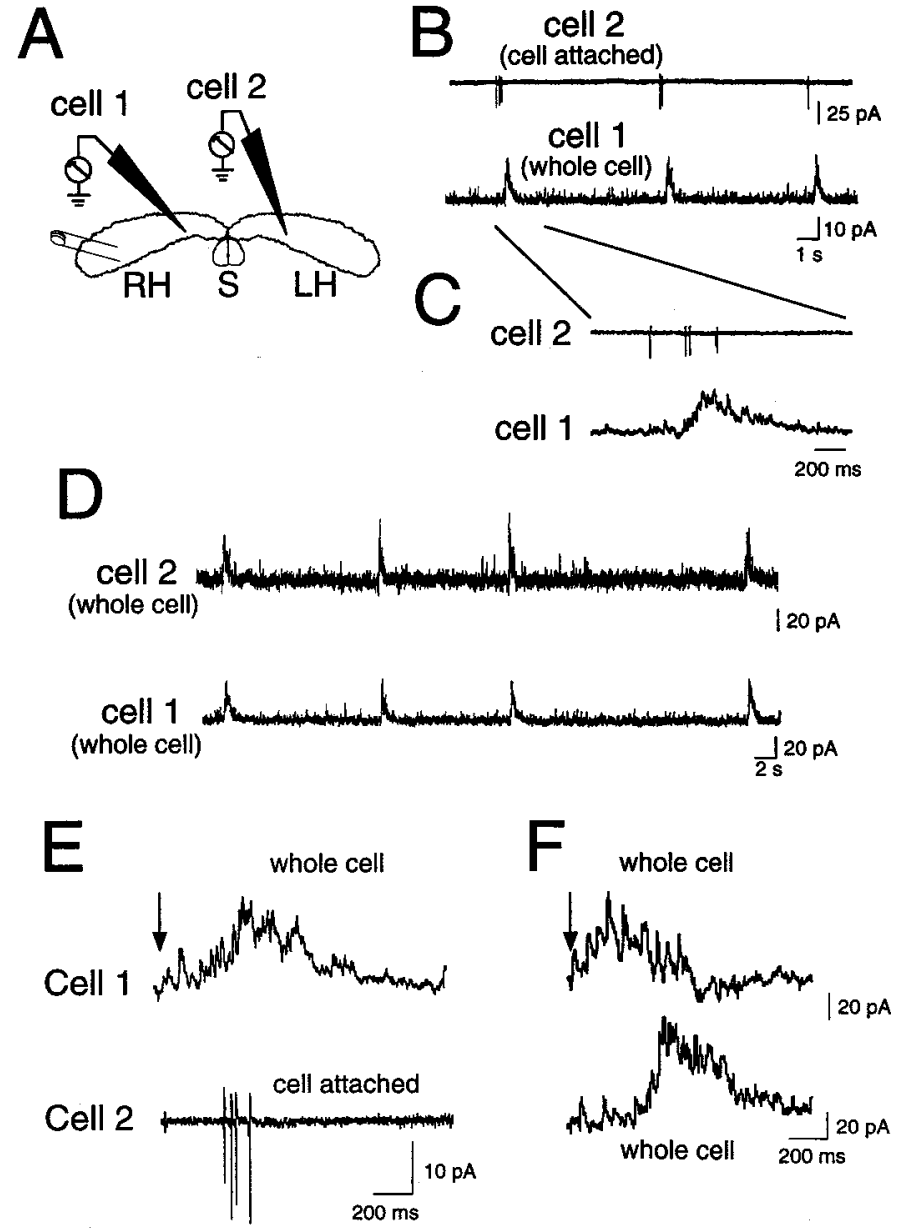

Figure 2. GDPs are synchronous in neurons from ipsilateral and contralateral hippocampi. Spontaneous $(B-D)$ and evoked $(E-F)$ GDPs recorded in the whole-cell configuration from two CA3 pyramidal cells ( $A$, one from each hippocampus). $B-C$, Simultaneous recording of two pyramidal cells in the cell-attached (cell 2) and whole-cell (cell 1) configurations. Note that bursts of action potentials occur in cell 2 during GDP in cell 1 (the first GDP of $B$ is presented in an expanded time scale in $C$ ). $D$, Entry into whole-cell mode in cell 2 shows that GDPs are synchronous in both cells. $E-F$, GDPs evoked in the same two cells as in $B-D$ by electrical stimulation ( $E$ : cell 1, whole-cell mode; cell 2, cell-attached mode; $F$ : both cells in whole-cell mode). Note that electrical stimulation in the temporal extremity of one hippocampus generated GDP in both ipsilateral (cell 1) and contralateral (cell 2) hippocampi, whereas when spontaneous GDPs appeared simultaneously in both cells, the GDP evoked in the ipsilateral side preceded the contralateral GDP.

septal poles but did not propagate until temporal extremities. Precise analysis indeed revealed that although almost all GDPs (97 $\pm 1 \% ; n=24$ hippocampi) recorded in the most $10-25 \%$ septal part propagated to the first half of hippocampus, an increasing number of them faded with distance along the temporal half. As illustrated in Figure 3D, among GDPs recorded in the $10-25 \%$ most septal part of hippocampus, $85 \pm 4 \%$ propagated to the rest $50-65 \%$ of hippocampus ( $n=22$ hippocampi), $77 \pm 7 \%$ to the rest $65-80 \%$ of hippocampus $(n=17)$, and $60 \pm 8 \%$ reached the rest $80-90 \%$ of hippocampus $(n=12)$. Analysis of the delays of appearance of GDPs at these different locations indicated that this septotemporal propagation occurred at a speed of $7.55 \pm 2 \mathrm{~mm} / \mathrm{sec}(n=11$ hippocampi). Although GDP propagation tended to slow down in the temporal part, no significant difference in speeds was observed as a function of the portion of hippocampus $(9.2 \pm 2,9.4 \pm 2,7.7 \pm 1$, and $5 \pm 2 \mathrm{~mm} / \mathrm{sec}$ in the first $10-50,50-65,65-75$, and $75-90 \%$ of hippocampus respectively; $n=11$ hippocampi; $p>0.7$ ).

Therefore, in the intact septohippocampal complex in vitro, most of hippocampal GDPs appear in the septal poles and propagate bilaterally toward temporal extremities.

\section{Septotemporal orientation of hippocampal activity}

Taking advantage of the in vitro preparation, we investigated the behavior of surgically isolated parts of hippocampus to examine whether the septotemporal pattern of GDP propagation is determined by particular differences between septal and temporal hippocampal poles or by a septotemporal gradient of activity along the longitudinal hippocampal axis. Removal of the septum ( $n=18$ with hippocampus and septum together; $n=14$ with hippocampus alone), separation of each hippocampus $(n=13$ with both hippocampi together; $n=12$ with only one isolated intact hippocampus), and more surprisingly, isolation of septal and temporal halves of hippocampus $(n=11$ experiments with recording from the same hippocampus before and after separation of septal from temporal halves) did not affect the septotemporal pattern of propagation of GDPs (Fig. 4), suggesting that it does not require the integrity of the septohippocampal complex. In the temporal half of hippocampus, $80 \pm 9 \%$ of GDPs appeared in the most septal recording site and propagated in the septotemporal direction, whereas $7 \pm 7 \%$ appeared in the middle of the preparation and propagated in both septal and temporal directions, $1 \pm 1 \%$ appeared in the most temporal recording site and propagated toward the septal pole, $2 \pm 2 \%$ appeared simultaneously in all recording sites, and $9 \pm 5 \%$ appeared in only one recording site and did not propagate. For the septal half, the corresponding values were $72 \pm 12,1 \pm 2,15 \pm 8,12 \pm 5$, and $0 \%$, respectively.

To test the hypothesis that the septal pole could drive the intact hippocampal network because of higher spontaneous activity, we compared the frequency of spontaneous GDPs in isolated portions of hippocampus. In the experiment illustrated in Figure 5, measuring spontaneous activity before and after the cutting of hippocampus into one-thirds revealed a real septotemporal gradient of GDP frequency, the most septal third having similar GDP frequency as the intact hippocampus, whereas this frequency decreased by $30 \%$ and $80 \%$ in the middle and temporal thirds, respectively. In nine experiments in which GDP frequency was compared in septal versus temporal hippocampal halves, GDPs had a frequency of $10.9 \pm 1 \mathrm{~min}^{-1}$ in the septal half and $8.9 \pm 2 \mathrm{~min}^{-1}$ in the temporal half of intact hippocampus. After a knife cut was made to isolate septal from temporal halves $(n=$ 9), GDP frequency remained unchanged in the septal part (11.4 \pm $2 \mathrm{~min}^{-1}$ ) but decreased by two to four times in the temporal half $\left(5.3 \pm 1 \mathrm{~min}^{-1} ; p<0.05\right)$ (Fig. 5D).

A possible additional factor for the preferential septotemporal orientation of GDP propagation is derived from the observation that spontaneous GDPs initiated in the septal poles had a higher rate of propagation to the rest of the hippocampus than spontaneous GDPs initiated in the temporal poles. From 17 experiments in which both types of GDPs were present, we observed that $75 \pm$ $6 \%$ of GDPs initiated in the septal pole propagated to the temporal pole, whereas $41 \pm 9 \%$ of GDPs initiated in the temporal pole propagated to the septal pole (data not shown; $p<0.01$ ).

Therefore, septotemporal initiation and propagation of GDPs is supported by at least two factors: (1) GDPs occur more frequently in the septal than in the temporal pole; and (2) GDPs 


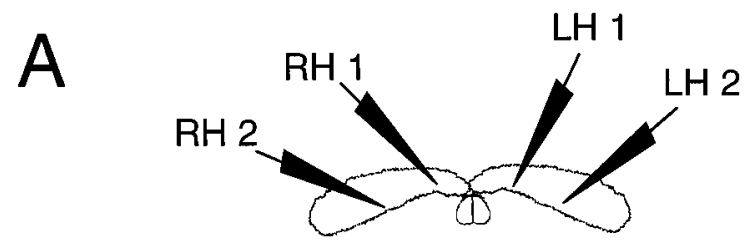

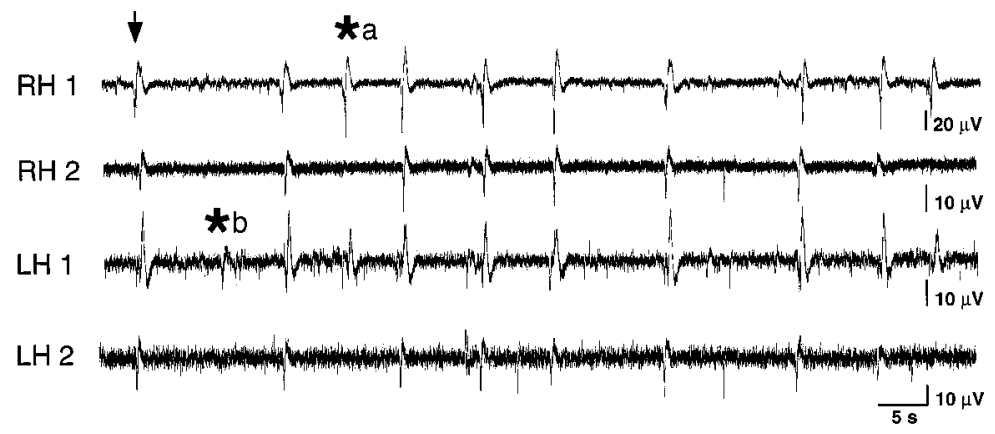
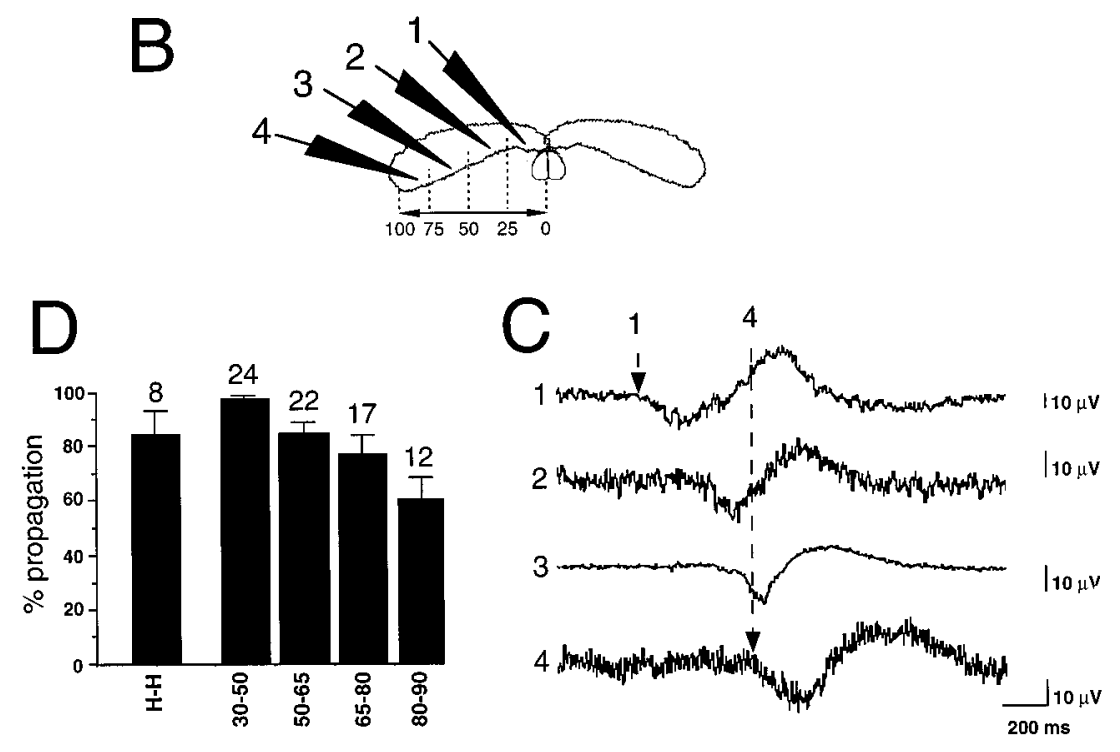

Figure 3. Spontaneous GDPs propagate synchronously in both hippocampi from septal to temporal poles. Multiple extracellular field recordings from the CA3 region of the intact bilateral septohippocampal complex. $A$, Simultaneous extracellular field recordings at the four recording sites indicated in the top scheme. $R H$, right hippocampus; $L H$, left hippocampus. Note that most GDPs (field deflections, arrow) appeared quite simultaneously in the four recording sites, with few exceptions of GDPs that appeared in septal poles but not in temporal poles (asterisk $a$ ) or appeared locally in one hippocampal site but did not propagate (asterisk $b$ ). $B-D$, Simultaneous extracellular field recordings at the four recording sites indicated in the scheme $(B)$. Corresponding electrophysiological traces (1-4) showing propagation of a GDP at a large time scale. Note that GDPs appeared first in the most septal recording site $(1$, dashed arrow) and propagated toward the most temporal recording site (4, dashed arrow). As illustrated in $A$, not all GDPs propagated to the temporal extremity or to the contralateral hippocampus. $D$, Summary of the percentage of GDP propagation from one hippocampus to its contralateral counterpart $(H-H)$ and from the septal pole to the other recording sites in the same hippocampus $(x$-axis values correspond to normalized septotemporal distances as represented in $B$ ). Numbers above each bar indicate the corresponding number of averaged experiments. Note that a significant percentage of GDPs initiated in the septal pole faded away before reaching the temporal extremity. initiated in the septal pole have a higher rate of propagation than GDPs initiated in the temporal pole. It remains to be elucidated whether this septotemporal gradient is attributable to differences in cell properties or to network organization, possibly in relation with maturational differences.

\section{GDPs in septum are driven by the activity of extrinsic hippocamposeptal projections}

To examine the relationship between septal and hippocampal GDPs, we recorded simultaneously the synaptic activity from septal and hippocampal neurons. As illustrated in Figure $6 A$, most GDPs $(92.5 \pm 4 \% ; n=4)$ appearing in the septal pole of the hippocampus appeared also in the medial septum. In addition, GDPs typically appeared first in the hippocampus before appearing in the septum (Fig. 6A).

To know whether GDPs could be generated in septum independently from hippocampus, we recorded synaptic activity from septal neurons before and after section of hippocamposeptal connections (Fig. 6). Although GDPs were present in septum when functionally connected to hippocampus, surgical isolation by a knife cut between hippocampus and septum abolished GDPs in septum but not in hippocampus $(n=12)$ (Fig. 6B). These results suggest that septal GDPs originate in hippocampus and propagate to septum via hippocamposeptal-projecting neurons.

Together, these results suggest that the septal pole of the hippocampus paces the activity of the developing intact septohippocampal complex in vitro.

\section{DISCUSSION}

Spontaneous neuronal activity of the intact neonatal septohippocampal complex in vitro was studied using simultaneous multiple whole-cell and extracellular field recordings. We found that spontaneous GDPs, initially described in hippocampal transverse slices (Ben-Ari et al., 1989), have particular spatiotemporal characteristics of generation and propagation in the intact septohippocampal complex. Three principal conclusions can be drawn from the present results: (1) the neonatal hippocampal network is characterized by a septotemporal gradient of automaticity; spontaneous GDPs are present in isolated transverse portions of hippocampus, but the septal pole has the highest rhythm of activity and drives the entire hippocampal network; (2) GDPs are synchronized in both hippocampi via commissural connections; and (3) in contrast to hippocampus, the septal network itself does 

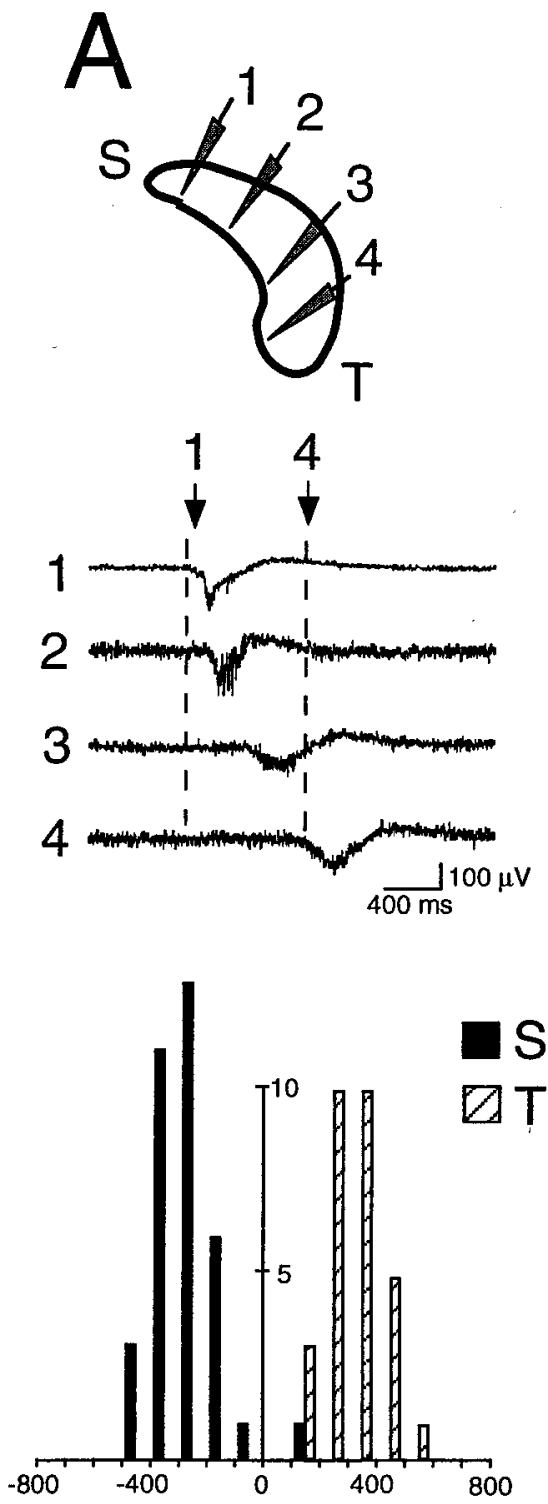
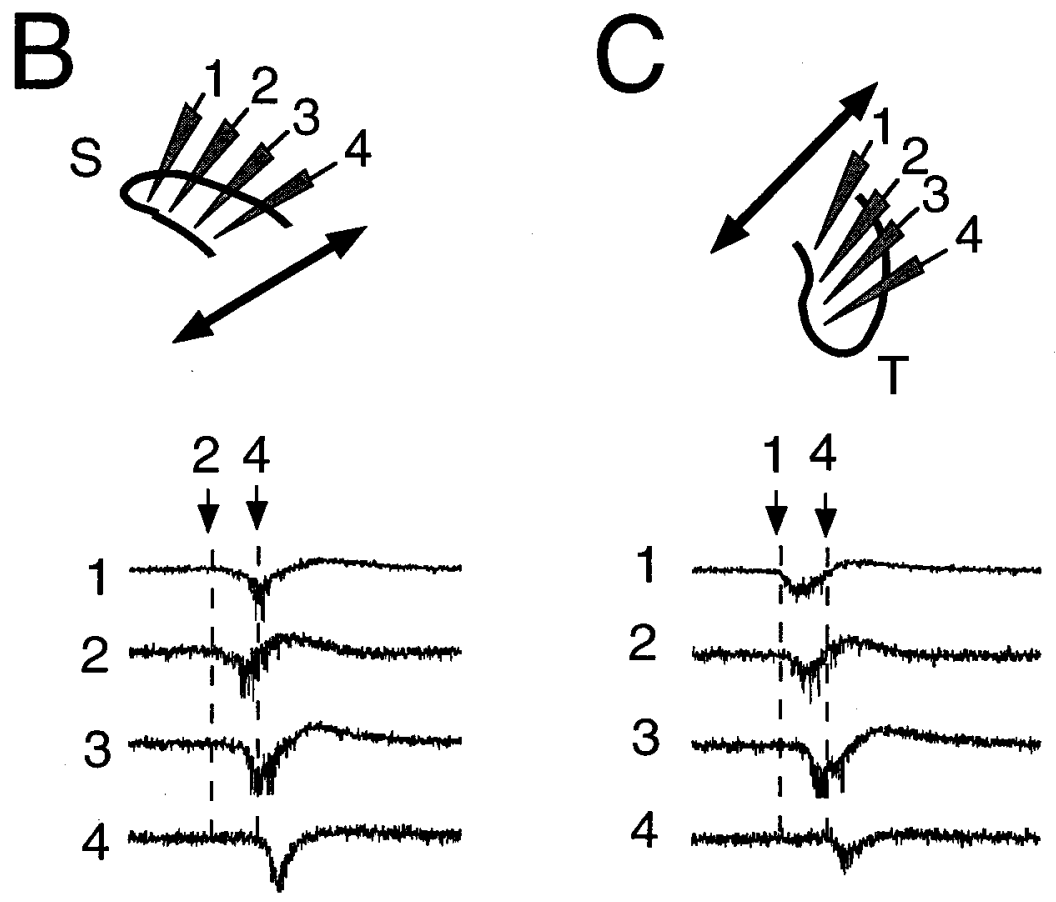

Figure 4. GDPs propagate in the septotemporal direction in isolated portions of hippocampus. Simultaneous recording of GDPs at different recording sites $(1-4)$ in the intact $(A)$, isolated septal $(B)$, or temporal $(C)$ halves of the hippocampus. Cross-correlograms for GDPs $(y$-axis, number of GDPs; $x$-axis, time, $100 \mathrm{msec}$ bin) of different recording sites (septal pole, filled bars; temporal pole, open bars) are plotted below the corresponding traces. $A$, Cross-correlogram of GDPs in sites 1 (septal pole, filled bars), 3 (reference event, GDP in site 3), and 4 (temporal pole, open bars) in the intact hippocampus. $B$, Cross-correlogram of GDPs in sites 1 (septal pole) and 4 (reference event, GDP in site 4 ) in the septal half of hippocampus. $C$, Cross-correlogram of GDPs in sites 1 (reference event, GDP in site 1) and 4 (temporal pole) in the temporal half of hippocampus. Note the septotemporal propagation of GDPs in the intact hippocampus, as well as in the septal and temporal halves of the same hippocampus after they were surgically transsected.

not generate GDPs. However, septal neurons undergo GDPs originating in and propagating from hippocampus. Therefore, the spontaneous neuronal activity of the intact septohippocampal complex is tightly synchronized during the first postnatal week of life, which may have major implications for the coordinated development of the neuronal ensembles in immature limbic structures.

\section{Septotemporal orientation of hippocampal activity}

Synchronized patterns of activity at the early stages of development were observed, primarily in the slice preparation, in various structures (O’Donovan et al., 1992; Yuste et al., 1992, 1995; Gu and Spitzer, 1995; Kandler and Katz, 1995; Feller et al., 1996; Mooney et al., 1996; Ben-Ari et al., 1997). The use of novel in vitro preparations such as the intact septohippocampal complex allows the study of the generation and propagation of network activities with similar technical facilities as in slices (Khalilov et al., 1997a,b; Leinekugel et al., 1997b). We recently showed that this preparation was fully viable and actually allowed better tissue preservation than age-matched slices (Khalilov et al., 1997a). Patch-clamp whole-cell recordings allow us to draw the conclusion that GDPs in the intact hippocampus have the same principal features as in hippocampal slices: (1) they are mainly GABAergic, because their reversal potential was primarily dependent on $\mathrm{Cl}^{-}$ gradient; and (2) they are locally synchronous, because they appeared synchronously in pairs of nearby individual cells.

However, recording from pairs of distant cells along the 

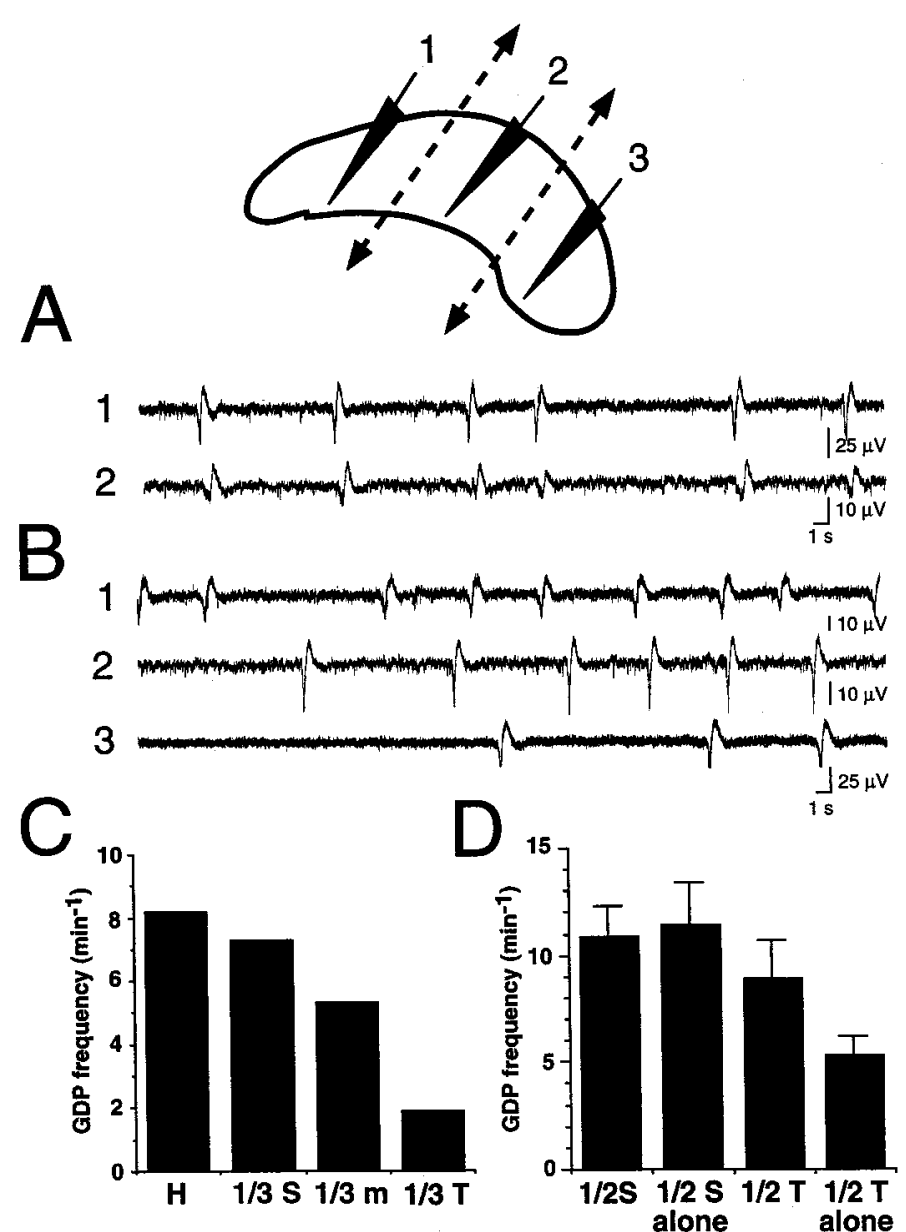

Figure 5. Septotemporal gradient of GDP frequency in the hippocampus. GDP frequency in different portions of hippocampus (top, recording sites 1-3) was measured before $(A)$ and after $(B)$ their surgical transsection (top, dashed lines). Note that in addition to being desynchronized after surgical transsection (compare $A, B$ ), GDP frequency gradually decreased toward the temporal pole (compare traces 1-3 in $B$ ). $C$, Quantification of GDP frequency before $(H)$ and after $(1 / 3 \mathrm{~S}$, most septal third; $1 / 3 \mathrm{~m}$, middle third: $1 / 3 \mathrm{~T}$, most temporal third) surgical transsection. $D$, Average GDP frequency ( $n=9$ hippocampi) in the septal and temporal halves before (1/2 $S$ and $1 / 2 T$, respectively) and after (1/2 $S$ alone and $1 / 2$ $T$ alone, respectively) surgical transsection. Note that whereas surgical hemisection did not affect GDP frequency in the septal half, it decreased GDP frequency by two times in the temporal half.

septotemporal axis, we observed systematic delays of GDP occurrence. Combining simultaneous whole-cell and multiple extracellular field-potential recordings clearly indicated that in the intact hippocampus, and more surprisingly also in isolated transverse portions of hippocampus, GDPs propagate in the septotemporal direction. These results suggest that the neonatal hippocampus has a septotemporal gradient of neuronal activity and/or a septotemporal orientation of neuronal projections.

Although most GDPs are initiated in the septal pole, a significant number of them are initiated in the temporal pole and are able to propagate toward the septal pole. Moreover, GDPs being initiated in the middle of hippocampus propagate toward both poles. These observations suggest that the hypothesis of asymmetric longitudinal orientation of projections is unlikely to fully explain the preferential septotemporal orientation of GDP propagation.
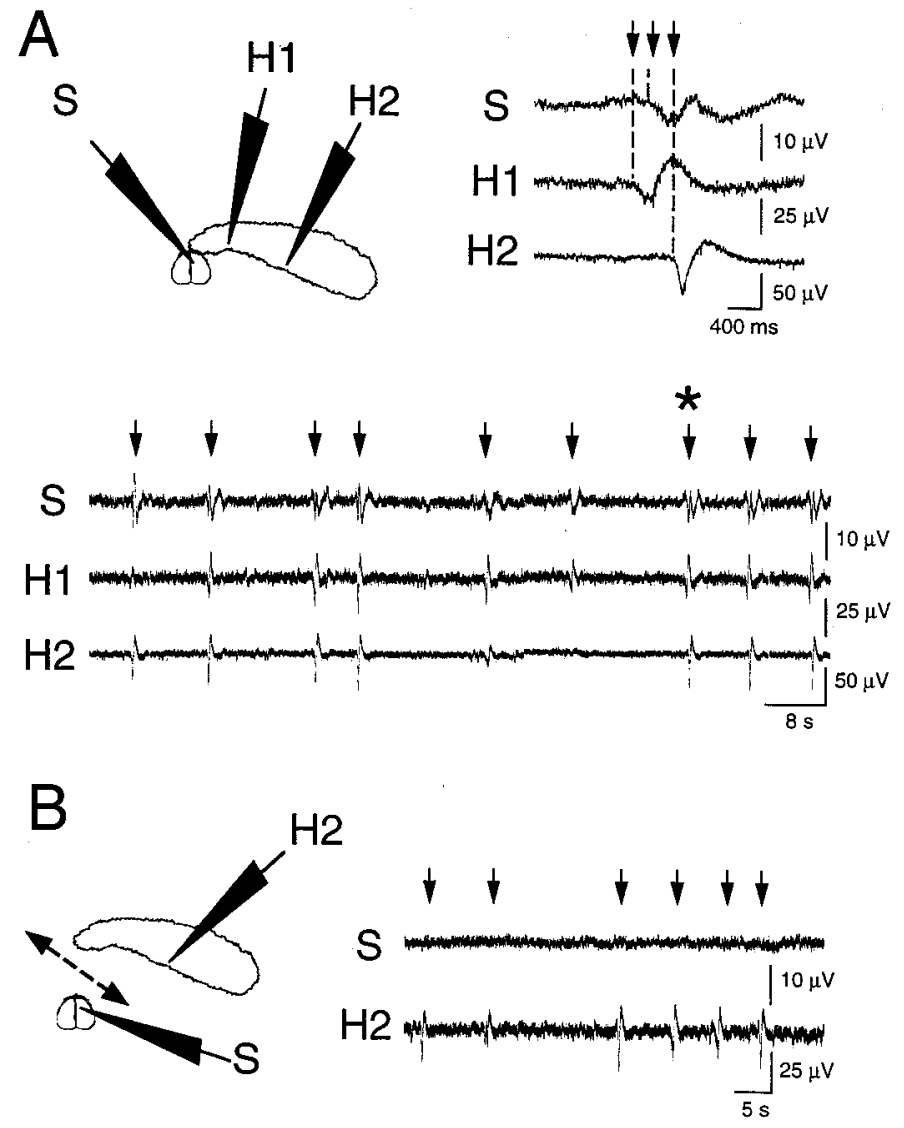

Figure 6. GDPs in medial septum originate in the hippocampus. Extracellular field potentials were recorded simultaneously from the medial septum (top scheme, $S$ ) and from the CA3 hippocampal region (top scheme, $H 1, H 2)$ before $(A)$ and after $(B)$ cutting the connections between hippocampus and septum $(B$, dashed line $)$. In the septohippocampal complex $(A)$, GDPs (arrows) were synchronized in the hippocampus and the medial septum. The GDP indicated by an asterisk in $A$ is presented in the right top corner at expended time scale, showing that GDPs typically appeared first in the septal pole of hippocampus $(H 1)$ and propagated to the septum $(S)$ and temporal pole of hippocampus $(H 2)$. After surgical separation of hippocampus and septum $(B)$, GDPs (arrows) were still present in hippocampus $(\mathrm{H} 2)$ but not in septum $(S)$.

Because GDPs are present in isolated portions of hippocampus at various septotemporal locations, including 500- $\mu$ m-thick transverse hippocampal slices and even minislices of CA3 subfield or fascia dentata (Khazipov et al., 1997), the neuronal elements required for their generation are present already in local neuronal circuits. However, the present results indicate a higher frequency of GDPs in isolated septal parts of hippocampus compared with more temporal parts. Therefore, the neonatal hippocampal network has a septotemporal gradient of autorhythmicity, and the septal pole, being the most active, paces the rhythm of the entire structure. Further investigations are required to examine whether this gradient is attributable to differences in cellular properties or in synaptic connectivity along the septotemporal axis of hippocampus. Because GABAergic interneurons play a major role in the generation of GDPs, an additional possibility is that an asymmetric longitudinal distribution of subclasses of GABAergic interneurons, as suggested in previous morphological studies (Buzsaki et al., 1990), could be implicated in the observed septotemporal gradient of GDPs frequency. 


\section{Synchronization of hippocamposeptal activity}

Our present results indicate that in the intact septohippocampal complex in vitro, GDPs are synchronous in both hippocampi and medial septum. However, they were absent in the isolated septum. Therefore, only the use of a preparation that preserves hippocamposeptal connections intact could allow the observation that GDPs represent a common pattern of activity for hippocampal and septal neurons, which is one of the major findings of this work.

Septal GDPs have some similarities with hippocampal GDPs, including their synchronous occurrence in neurons and the main contribution of $\mathrm{GABA}_{\mathrm{A}}$ receptors. However, the mechanisms of generation of GDPs in septum are different from in hippocampus. Although hippocampal GDPs can be generated in isolated portions of the hippocampal network, the local septal neuronal network does not seem able to generate GDPs. This may be attributable to the absence of glutamatergic recurrent collaterals in the septum (Jakab and Leranth, 1995), which play an important role in synergy with GABAergic excitatory connections in the generation of hippocampal GDPs (Ben-Ari et al., 1997; Khazipov et al., 1997; Leinekugel et al., 1997a). Instead, septal GDPs result from the propagation of hippocampal GDPs via hippocamposeptal projections.

From previous morphological and electrophysiological studies (Freund and Antal, 1988; Buzsaki et al., 1992; Toth et al., 1993; Lee et al., 1994; Bragin et al., 1995; Buzsaki and Chrobak, 1995; Ylinen et al., 1995; Freund and Buzsaki, 1996; Toth et al., 1997), extensive GABAergic networks were proposed to play a key role in the synchronization of limbic neurons in the adult hippocampus, septum, and entorhinal cortex, giving rise to spontaneous patterns of synchronized activities (theta rhythm, sharp waves). Several morphological studies using retrograde and anterograde labeling of hippocamposeptal and interhippocampal (commissural) neuronal projections have already reported the presence of early GABAergic connections (Crutcher, 1982; Milner et al., 1983; Linke and Frotscher, 1993; Super and Soriano, 1994). The present results suggest that hippocamposeptal and commissural projections are already functional at birth and support the view of a major role of the GABAergic network in synchronizing activity of large neuronal ensembles, inside and across developing limbic structures.

\section{Possible physiological implications of GDPs propagation}

Previous works on hippocampal slices showed that GDPs were associated with synchronous $\mathrm{Ca}^{2+}$ oscillations mediated by voltage-dependent $\mathrm{Ca}^{2+}$ channels and NMDA receptors (Leinekugel et al., 1996, 1997a). Although the consequences of the $\mathrm{Ca}^{2+}$ influx during GDPs via voltage-gated $\mathrm{Ca}^{2+}$ channels and NMDA channels are presently unknown, activation of voltage-gated $\mathrm{Ca}^{2+}$ channels promotes neuronal differentiation in several preparations (Desarmenien and Spitzer, 1991; Gu and Spitzer, 1995; LoTurco et al., 1995; Rusanescu et al., 1995), and localized $\mathrm{Ca}^{2+}$ influx through NMDA receptors can provide a hebbian modulation of developing synapses (Komatsu and Iwakiri, 1993; Crair and Malenka, 1995; Fox, 1995; Kirkwood et al., 1995; Durand et al., 1996; Liao and Manilow, 1996; McLean et al., 1996; Wu et al., 1996). It is now generally accepted that the establishment and modulation of synaptic connections during development is at least partly dependent on the coordination between presynaptic and postsynaptic neuronal elements (Constantine-Paton et al., 1990; Goodman and Shatz, 1993; Katz and Shatz, 1996). It was shown recently that depending on the precise timing of presynaptic and postsynaptic firing, either longterm potentiation or long-term depression of synaptic transmission could be induced (Markram et al., 1997). Interestingly, GDPs provided high synchronization locally, but because of their slow speed of propagation, distant cells in the septotemporal hippocampal axis have very significant delays of activation (up to $1 \mathrm{sec})$. According to the hebbian hypothesis "neurons that fire together, wire together" (Stent, 1973), local synchrony could serve as a basis for the formation of functional units inside large neuronal ensembles. Existence of functional units in the septohippocampal system was recently suggested from morphological studies indicating that distinct groups of cells, depending on their positions along the longitudinal axis of hippocampus, project to distinct specific areas of septum (Risold and Swanson, 1996). One may therefore assume that GDPs, by differentially regulating synaptic efficacy between neurons depending on their septotemporal distance, may drive the formation of functional units in the septohippocampal complex.

Although the intact septohippocampal preparation in vitro allows us to characterize the neuronal activity in intact structures, further studies are needed to examine whether similar spatiotemporal characteristics of spontaneous activity are present in the neonatal limbic system in vivo.

\section{REFERENCES}

Ben-Ari Y, Cherubini E, Corradetti R, Gaïarsa JL (1989) Giant synaptic potentials in immature rat CA3 hippocampal neurones. J Physiol (Lond) 416:303-325.

Ben-Ari Y, Khazipov R, Leinekugel X, Caillard O, Gaïarsa JL (1997) $\mathrm{GABA}_{\mathrm{A}}, \mathrm{NMDA}$ and AMPA receptors: a developmentally regulated "ménage à trois." Trends Neurosci 20:523-529.

Blanton M, LoTurco J, Kriegstein A (1989) : Whole cell recording from neurons in slices of reptilian and mammalian cerebral cortex. J Neurosci Methods 30:203-210.

Bragin A, Jando G, Nadasdy Z, Hetke J, Wise K, Buzsaki G (1995) Gamma (40-100 Hz) oscillation in the hippocampus of the behaving rat. J Neurosci 15:47-60.

Buzsaki G, Chrobak JJ (1995) Temporal structure in spatially organized neuronal ensembles: a role for interneuronal networks. Curr Opin Neurobiol 5:504-510.

Buzsaki G, Chen L, Gage F (1990) Spatial organization of physiological activity in the hippocampal region: relevance to memory formation. Prog Brain Res 83:257-267.

Buzsaki G, Horvath Z, Urioste R, Hetke J, Wise K (1992) Highfrequency network oscillation in the hippocampus. Science 256:1025-1027.

Constantine-Paton M, Cline HT, Debski E (1990) Patterned activity, synaptic convergence, and the NMDA receptor in developing visual pathways. Annu Rev Neurosci 13:129-154.

Corradetti R, Gaïarsa JL, Ben-Ari Y (1988) D-Aminophosphonovaleric acid-sensitive spontaneous giant EPSPs in immature rat hippocampal neurones. Eur J Pharmacol 154:221-222.

Crair MC, Malenka RC (1995) A critical period for long-term potentiation at thalamocortical synapses. Nature 375:325-328.

Crutcher KA (1982) Development of the rat septohippocampal projection: a retrograde fluorescent tracer study. Dev Brain Res 3:145-150.

Desarmenien MG, Spitzer NC (1991) Role of calcium and protein kinase $\mathrm{C}$ in development of the delayed rectifier potassium current in Xenopus spinal neurons. Neuron 7:797-805.

Durand GM, Kovalchuk Y, Konnerth A (1996) Long-term potentiation and functional synapse induction in developing hippocampus. Nature 381:71-75.

Feller MB, Wellis DP, Stellwagen D, Werblin FS, Shatz CJ (1996) Requirement for cholinergic synaptic transmission in the propagation of spontaneous retinal waves. Science 272:1182-1187.

Fox K (1995) The critical period for long-term potentiation in primary sensory cortex. Neuron 15:485-488.

Freund TF, Antal M (1988) Gaba-containing neurons in the septum control inhibitory interneurons in the hippocampus. Nature 336:170-172. 
Freund TF, Buzsaki G (1996) Interneurons of the hippocampus. Hippocampus 6:347-470.

Gaïarsa JL, Corradetti R, Cherubini E, Ben-Ari Y (1990) Modulation of GABA-mediated synaptic potentials by glutamatergic agonists in neonatal CA3 rat hippocampal neurons. Eur J Neurosci 3:301-309.

Goodman CS, Shatz CJ (1993) Developmental mechanisms that generate precise patterns of neuronal connectivity. Cell 72:77-98.

Gu X, Spitzer NC (1995) Distinct aspects of neuronal differentiation encoded by frequency of spontaneous $\mathrm{Ca}^{2+}$ transients. Nature 375:784-787.

Jakab RL, Leranth C (1995) Septum. In: The rat nervous system, p 405-442. San Diego: Academic.

Kandler K, Katz LC (1995) Neuronal coupling and uncoupling in the developing nervous system. Curr Opin Neurobiol 5:98-105.

Katz LC, Schatz CJ (1996) Synaptic activity and the construction of cortical circuits. Science 274:1133-1138.

Khalilov I, Esclapez M, Medina I, Aggoun D, Lamsa K, Leinekugel X, Khazipov R, Ben-Ari Y (1997a) A novel in vitro preparation: the intact hippocampal formation. Neuron 19:743-749.

Khalilov I, Lamsa K, Khazipov R, Leinekugel X, Medina I, AggounZaoui J, Esclapez M, Ben-Ari Y (1997b) Epileptiform activity in intact hippocampus in vitro. Soc Neurosci Abstr 27:2106.

Khazipov R, Leinekugel X, Khalilov I, Gaïarsa JL, Ben-Ari Y (1997) Synchronization of GABAergic interneuronal network in CA3 subfield of neonatal hippocampal slices. J Physiol (Lond) 498:763-772.

Kirkwood A, Lee HK, Bear MF (1995) Co-regulation of long-term potentiation and experience-dependent synaptic plasticity in visual cortex by age and experience. Nature 375:328-331.

Komatsu Y, Iwakiri M (1993) Long-term modification of inhibitory synaptic transmission in developing visual cortex. NeuroReport 4:907-910.

Lee MG, Chrobak JJ, Sik A, Wiley RG, Buzsaki G (1994) Hippocampal theta activity following selective lesion of the septal cholinergic system. Neuroscience 62:1033-1047.

Leinekugel X, Tseeb V, Ben-Ari Y, Bregestovski P (1995) Synaptic $\mathrm{GABA}_{\mathrm{A}}$ activation induces $\mathrm{Ca}++$ rise in pyramidal cells and interneurons from rat neonatal hippocampal slices. J Physiol (Lond) 487:319-329.

Leinekugel X, Khazipov R, Medina I, Khalilov I, Represa A, Ben-Ari Y (1996) Synchronous $\mathrm{Ca}^{2+}$ oscillations controlled by the synergistic actions of $\mathrm{GABA}_{\mathrm{A}}$ and NMDA receptors in the neonatal rat hippocampus. Soc Neurosci Abstr 26:221.

Leinekugel X, Medina I, Khalilov I, Ben-Ari Y, Khazipov R (1997a) $\mathrm{Ca}^{2+}$ oscillations mediated by the synergistic excitatory actions of $\mathrm{GABA}_{\mathrm{A}}$ and NMDA receptors in the neonatal hippocampus. Neuron 18:243-255.

Leinekugel X, Khazipov R, Khalilov I, Tremblay E, Ben-Ari Y (1997b) Giant depolarizing potentials in the neonatal rat intact hippocampus in vitro. Soc Neurosci Abstr 27:2106.

Leinekugel X, Khalilov I, McLean H, Caillard O, Gaïarsa JL, Ben-Ari Y, Khazipov R (1998) GABA is the principal fast acting excitatory transmitter in the neonatal hippocampus. In: Basic mechanisms of the epilepsies (Jasper E, ed). Hagerstown, MD: Lippincott-Raven.
Liao D, Malinow R (1996) Deficiency in induction but not expression of LTP in hippocampal slices from young rats. In: Learning and memory, pp 138-149. Cold Spring Harbor, NY: Cold Spring Harbor.

Linke R, Frotscher M (1993) Development of the rat septohippocampal projection: tracing with DiI and electron microscopy of identified growth cones. J Comp Neurol 332:69-88.

LoTurco JJ, Owens DF, Heath MJS, Davis MBE, Kriegstein AR (1995) GABA and glutamate depolarize cortical progenitor cells and inhibit DNA synthesis. Neuron 15:1287-1298.

Markram H, Lübke J, Frotscher M, Sakmann B (1997) Regulation of synaptic efficacy by coincidence of postsynaptic APs and EPSPs. Science 275:213-215.

McLean HA, Caillard O, Ben-Ari Y, Gaïarsa JL (1996) Bidirectional plasticity expressed by GABAergic synapses in the neonatal rat hippocampus. J Physiol (Lond) 496:471-477.

Milner TA, Loy R, Amaral DG (1983) An anatomical study of the development of the septo-hippocampal projection in the rat. Dev Brain Res 8:343-371.

Mooney R, Penn AA, Gallego R, Shatz CJ (1996) Thalamic relay of spontaneous retinal activity prior to vision. Neuron 17:863-874.

O'Donovan MJ, Sernagor E, Sholomenko G, Ho S, Antal M, Yee W (1992) Development of spinal motor networks in the chick embryo. J Exp Zool 261:261-273.

Risold P, Swanson L (1996) : Structural evidence for functional domains in the rat hippocampus. Science 272:1484-1486.

Rusanescu G, Qi H, Thomas SM, Brugge JS, Halegoua S (1995) Calcium influx induces neurite growth through a Src-Ras signalling cassette. Neuron 15:1415-1425.

Stent G (1973) : A physiological mechanism for Hebb's postulate of learning. Proc Natl Acad Sci USA 70:997-1001.

Super H, Soriano EJ (1994) The organization of the embryonic and early postnatal murine hippocampus. II. Development of entorhinal, commissural, and septal connections studied with the lipophilic tracer DiI. J Comp Neurol 344:101-120.

Toth K, Borhegyi Z, Freund T (1993) Postsynaptic targets of GABAergic hippocampal neurons in the medial septum-diagonal band of Broca complex. J Neurosci 19:3712-3724.

Toth K, Freund TF, Miles R (1997) Disinhibition of rat hippocampal pyramidal cells by GABAergic afferents from the septum. J Physiol (Lond) 500:463-474.

Wu GY, Malinow R, Cline HT (1996) Maturation of a central glutamatergic synapse. Science 274:972-976.

Ylinen A, Soltesz I, Bragin A, Penttonen M, Sik A, Buzsaki G (1995) Intracellular correlates of hippocampal theta rhythm in identified pyramidal cells, granule cells and basket cells. Hippocampus 5:78-90.

Yuste R, Peinado A, Katz LC (1992) Neuronal domains in developing neocortex. Science 257:665-669.

Yuste R, Nelson DA, Rubin WW, Katz LC (1995) Neuronal domains in developing neocortex: mechanisms of coactivation. Neuron 14:7-17. 